International Journal of MCH and AIDS (2019), Volume 8, Issue I, 44-53

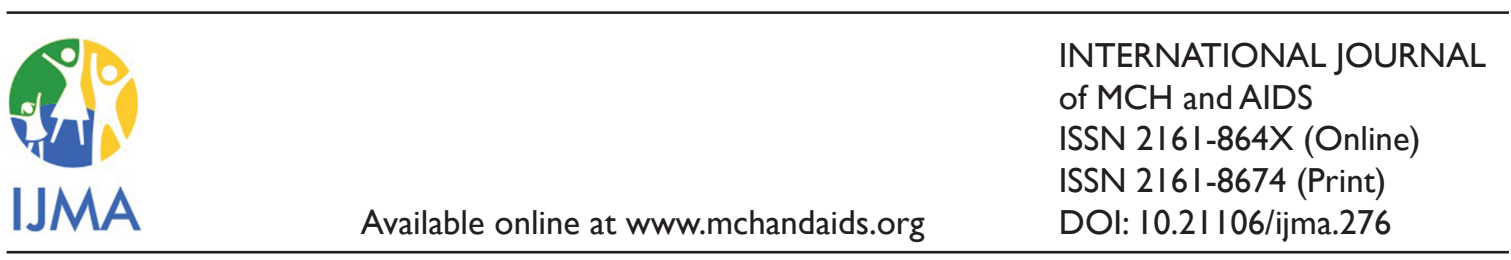

ORIGINAL ARTICLE

\title{
Progress and Challenges of Implementing Decentralized HIV Testing For Prevention of Mother-to-Child Transmission of HIV - Myanmar
}

\author{
San Hone, PhD; ${ }^{1,2}$ Li Li, PhD; ${ }^{3}$ Sung-Jae Lee, PhD; ${ }^{4}$ W. Scott Comulada, PhD; ${ }^{5}$ Roger Detels, MD, MS ${ }^{6}$ \\ 'Department of Epidemiology, 7I-269 CHS, UCLA School of Public Health, Los Angeles CA USA; ${ }^{2}$ National AIDS Program, Ministry of Health and Sports, \\ Nay Pyi Taw, Myanmar; ${ }^{3}$ Department of Psychiatry and Biobehavioral Sciences, Semel Institute for Neuroscience and Human Behavior, Department of \\ Epidemiology, and UCLA Fielding School of Public Health, 10920 Wilshire Blvd., Suite 350; Los Angeles, CA 90024, USA; ${ }^{4}$ Department of Psychiatry \\ \& Biobehavioral Sciences, Center for Community Health, and UCLA Fielding School of Public Health, I0920 Wilshire Blvd., Suite 350; Los Angeles, \\ CA 90024, USA; ${ }^{5}$ Department of Psychiatry and Biobehavioral Sciences, and UCLA Center for Community Health, Los Angeles, CA, USA; ${ }^{6}$ Department \\ of Epidemiology, 7I-269 CHS, UCLA Fielding School of Public Health, Los Angeles CA, USA \\ Corresponding author email: detels@ucla.edu
}

ABSTRACT

Background: Myanmar has adopted point-of-care (POC) HIV testing for its prevention of mother-to-child transmission of HIV program, and was initiated in 84 townships in 2013. This study assessed the progress of HIV testing uptake from 20I2, one year prior to POC testing, to 20I5, and the challenges faced by service providers during the rapid rollout of this testing strategy.

Methods:This serial cross-sectional study included 23 townships randomly selected from the 84 townships. An open-question survey was used to collect information on the challenges faced by service providers. A random effects logistic model was used for assessing the progress of HIV testing uptake among urban and rural health center groups.

Results: HIV testing uptake for antenatal care (ANC) attendees increased from $60 \%$ to $90 \%$ for rural and from $70 \%$ to $90 \%$ for urban attendees. The proportion of ANC attendees who were tested at their first visit increased from $70 \%$ to $80 \%$ for rural and from $70 \%$ to $90 \%$ for urban attendees. In addition, the proportion receiving same-day test results increased from less than $10 \%$ to $90 \%$ for both groups. Major challenges faced during the initial rollout included low health awareness among pregnant women, fear of stigma and discrimination, long travel times and costs, and increased workloads of providers in rural settings.

Conclusions and Global Health Implications: The program should consider recruiting local volunteers to help reduce the workloads of service providers. Professional education based on need and continued mentoring and quality control schemes for HIV testing need to be in place. This decentralized strategy would be applicable to other resource-limited countries.

Key words: HIVIAIDS • Pregnant Women • Point-of-Care (POC) HIV Testing • Antenatal Care (ANC)

- Prevention of Mother-to-Child Transmission of HIV (PMCT) • Service Cascade • Stigma and Discrimination

Copyright (C) 2019 Hone et al.This is an open-access article distributed under the terms of the Creative Commons Attribution License, which permits unrestricted use, distribution, and reproduction in any medium, provided the original work is properly cited. 


\section{Introduction}

\section{I. Background of the study}

Acknowledging the feminizing pattern of the HIV epidemic, Myanmar has prioritized prevention of mother-to-child transmission (PMCT) as one of the most important interventions since early 2000. Accordingly, the PMCT program, which was pilottested in a few major cross-border townships in 200 I, gradually expanded each year to cover more geographic areas. By the end of 2015, the program reached $30 \mathrm{I}$ of the 330 townships in Myanmar and 38 hospitals (including 10 of the 38 tertiary level referral hospitals and 28 of the 40 secondary level referral hospitals).'

HIV testing is the entry point for identifying pregnant women living with HIV and enrollment for receiving PMCT services. Until 20I2, HIV testing was conducted according to the national HIV testing strategies, which allowed testing to be performed at health facility laboratories where there was at least one trained lab technician. This stringent testing guideline hindered practical scaling up of the PMCT program. Test results were not always available on the same day, depending on the workloads of the labs. ${ }^{2}$ This meant that pregnant women in both urban and rural areas usually got their results during subsequent $A N C$ visits instead of on the same day.

Consequently, the increase in coverage of the PMCT program did not meet the program's expectations for proportionately increased uptake of HIV testing and higher percentages of pregnant women who received their test results. ${ }^{2}$ Only an estimated $25-30 \%$ of pregnant women were tested and received their results through the PMCT program. ${ }^{3}$ In 2012, only half of the ANC attendees who received pre-test counselling were tested and received their test results. ${ }^{4}$ The high dropout rates between pre- and post-testing receipt of results became the main challenge for scaling up the program, especially since Myanmar's PMCT regimen was continuously changing to adopt updated World Health Organization's (WHO) recommendations on treating HIV-infected pregnant women as early as possible. ${ }^{5}$ Myanmar has pledged to achieve the global goal of eliminating paediatric HIV infection by
$2020,{ }^{6}$ which would require at least $95 \%$ HIV testing coverage of ANC attendees as a major process indicator. $^{7}$

Considering the barriers against achieving the global target of HIV testing coverage and provision of ART to all HIV-infected pregnant women as early as possible, Myanmar rolled out the innovative point-of-care (POC) testing strategy for the PMCT program in late 2013. It was initiated in 84 PMCT townships in 2013, an additional 44 townships in 2014 , and the remaining 173 townships in 2015 .

For POC testing, midwives were trained to use a simple, rapid diagnostic test kit for HIV testing according to the providers' guidelines, using a clear standard operational procedure in the local language. ${ }^{8}$ With the POC diagnosis, midwives were allowed to provide a screening test for all pregnant women seeking antenatal care (ANC). The screening test reactive individuals or specimens need to be referred or send to township hospital laboratories or sexually transmitted disease (STD) clinic laboratories for confirmation testing.

\section{I.2. Objectives/Specific aims}

With the rollout of POC testing, it is important to evaluate the progress of HIV testing coverage and timeliness of testing and result provision during the first two years of the program's implementation. As $70 \%$ of Myanmar's population resides in rural areas, ${ }^{9}$ it is important to compare urban and rural status. In addition, it is crucial to explore the major challenges experienced in townships while implementing POC testing so that remedial actions can be put in place for scaling up in future. Findings from this study will be useful for future program improvements to meet the global goal of eliminating paediatric HIV infection by $2020 .^{10}$

\section{Methods}

\section{I. Study design}

This was a mixed-methods study conducted in 23 townships randomly selected by a random number generator" from a list of townships where POC testing was rolled out in 2013. The quantitative component (serial cross-sectional study) assessed 
the progress of HIV testing coverage among pregnant women, followed by a qualitative study exploring challenges faced by primary care providers rendering POC HIV testing and linkage to care.

ANC facilities (health centers) in the selected townships were serially assessed from 2012 to 2015 to provide quantitative data. Qualitative data was collected from primary health care providers from each of the selected townships who were voluntarily recruited during the 2016 data collection period of January to September.

\subsection{Data collection}

\subsection{Health center data}

Information on the performance of HIV testing coverage and time of receiving test results were extracted from existing reports and records. Data entry was done using Epi Info ${ }^{\mathrm{TM}},{ }^{12}$ cross-checking with double data entry process to assure data quality.

\subsubsection{Care provider data}

A confidential self-administered semi-structured open-question survey was used to collect information from health care providers about attendees' gestational weeks at first ANC, perceptions of providing $\mathrm{POC}$ testing in terms of workload and difficulties in providing HIV testing at first ANC visit, challenges of referring HIV-positive pregnant women for confirmation testing, and suggestions for better program performance in the future.

\subsection{Data analysis}

\subsection{Health center data}

Prior knowledge of differences in health care management between facilities in urban and rural settings led to classifying the facilities as either urban or rural and comparing the outcomes over time. To detect changes in 2013 when POC testing was rolled out in the last quarter, 2012 annual data were used as a baseline, and annual data for 2013,2014, and 2015 were used as time points I, 2 , and 3 , respectively.

The major outcomes of interest included the proportion of ANC attendees receiving HIV testing and results, and among those tested, the proportion receiving HIV testing at the first $A N C$ and receiving same-day test results. These outcome variables were dichotomized as high or low, using the cut-off points based on the median values of particular outcomes across the study period. The cut-off was $80 \%$ for receiving HIV testing and results and receiving HIV testing at first ANC visit, and $70 \%$ for receiving same-day results.

Information for outcomes of interest was repeatedly assessed for four consecutive calendar years (20I 2 to 20I5) for all ANC facilities in selected townships. Observations were linked over time at the township level.The Proc GLIMMIX procedure in SAS version 9.4 was used for random effect logistic regression with the Gaussian quadrature method for modeling changes in outcomes of interest over time. ${ }^{13}$

The possible mean structures over time for each of the three major outcomes were assessed by plotting the mean logit scores for observing high proportions. All graphs showed non-linear trends over time. Among all possible non-linear mean structures, the one with the lowest AIC and QIC values (i.e., spline (knot at time 2) model) was selected as the final model.

The spline model estimates the outcome of interest in 2 segments of time: from baseline to time point 2 , and after time point 2 . The model used two time covariates, linear and spline time, each of which represents a specific time segment. The linear time covariate reveals the trends of the reference group from baseline to time point 2 , and the summation of linear time and spline time reveals the trends after time point 2. The interaction covariates (linear time*rural) reveal the differences in trends between reference (urban) and rural group from baseline to time point 2 , whereas (spline time*rural) reveals the differences in trends after time point 2 . The possible confounding effects of external variables on HIV testing uptake and those receiving ART were considered and controlled for in the analysis.

\subsubsection{Care provider data}

Responses for each of the semi-structured questionnaires enquiring about challenges faced by care providers while providing POC HIV testing services 
for pregnant women were independently coded by the principal investigator, and thematic analyses were conducted after several iterations, yielding seven distinct challenges. The findings were given in simple descriptions under each theme/challenge.

\section{Results}

All ANC facilities in the selected 23 townships participated in this study. Based on geographic location, these facilities were classified as urban or rural ANC facilities, which totalled $3 \mathrm{I}$ urban clinics and $\mathrm{I} 8 \mathrm{I}$ rural clinics.Among 960 primary health care providers, 647 voluntarily provided information via a confidential open questionnaire, resulting in an overall response rate of $67 \%$.

\section{I. Proportion of ANC attendees who received HIV testing and test results}

At baseline, half of the rural facilities had $60 \%$ HIV testing coverage among ANC attendees, whereas half of the urban facilities had $80 \%$ coverage. At the end of the study period, about half of rural and urban facilities had 100\% coverage (Table I).

As seen in the model output (Table 2), rural facilities had significantly lower log odds of $80 \%$ testing uptake than urban facilities at baseline. Both groups showed increasing log odds over time. The annual rate of improvement from baseline up to time point 2 was higher in the rural group ( $P$ value $<0 . I)$, and the rate of increase after time point 2 became lower in the rural group and higher in the urban group ( $P$ value $>0$. I). The trends of increase for both groups were depicted regarding probability in Figure $\mathrm{I}$.

Among the possible effects of external factors, health center groups with female literacy rates of 93\% and less had significantly better HIV testing uptake rates.

\subsection{Proportion of ANC attendees being tested at their first visit}

Although it would be preferable to assess the progress of HIV testing in terms of specific gestational week (before 14 week), it was very cumbersome for providers to specify exact gestational week at the time of testing, due to the inefficient paper-based registration system. Health centers usually prepare and keep ANC reports for first and subsequent visits, but do not record gestational week of ANC attendance. We could therefore only extract HIV testing coverage at the first ANC rather than at specific gestational week.

Phase of pregnancy at which women seek ANC was queried in the survey of health care providers. About one-third of providers said ANC commenced during the first trimester (before 14 weeks of gestation), half said that the first ANC visit usually took place during the second trimester (14 to 28 weeks), and the remaining $(\sim 15 \%)$ said the first ANC took place only after the second trimester.

The average HIV testing coverage at first ANC visit was $70 \%$ for both rural and urban centers at the baseline, then increased to $80 \%$ for rural and $90 \%$ for urban at the end of the study period (Table I).

According to model output (Table 2), the urban group showed a 0.6 annual increase in log odds for achieving $80 \%$ HIV testing for the first ANC attendees until time point 2 , and a slower rate of increase from time point 2 to 3 . The rural centers had lower log odds at baseline, which remained at the same level until time point 2 , but increased 0.9 between time points 2 and 3 . None of the rates of increase were statistically significant. The trends in the probability of achieving $80 \%$ HIV testing for the first ANC attendees for urban and rural centers are shown in Figure I.

\subsection{Proportion who received their results on the same day as testing}

The third outcome of interest was whether POC testing could shorten the test result turnaround time. The average level of providing same-day HIV test result was $0 \%$ for rural and $10 \%$ for clinics at the baseline, which increased to $90 \%$ for both groups at the end of the study (Table I).

Urban centers showed increasing log odds of providing test results on the same day, with an increase of $4.48 \mathrm{log}$ odds per year up to time point 2 (P-value $<0.01$ ), then increases of 3.01 (i.e., summation of coefficients for linear and spline time terms $=4.48$ I.47) log odds each year after time point 2. Rural centers had significantly lower log odds than urban 
Table I: Progress along the cascade of PMCT services by rural and urban ANC facility groups in Myanmar (20I2-20I5)

\begin{tabular}{|c|c|c|c|c|c|c|}
\hline \multirow[t]{2}{*}{ Description } & \multicolumn{3}{|c|}{ Rural } & \multicolumn{3}{|c|}{ Urban } \\
\hline & Median & Mean & $95 \% \mathrm{CL}$ & Median & Mean & 95\% CL \\
\hline \multicolumn{7}{|c|}{ Proportion of estimated number of pregnant women receiving ANC } \\
\hline Baseline & 0.7 & 0.7 & $(0.7 ; 0.8)$ & 0.8 & 0.8 & $(0.7 ; 0.9)$ \\
\hline Time point I & 0.8 & 0.8 & $(0.7 ; 0.8)$ & 0.8 & 0.8 & $(0.7 ; 0.9)$ \\
\hline Time point 2 & 0.8 & 0.8 & $(0.8 ; 0.9)$ & 0.9 & 0.8 & $(0.7 ; 0.9)$ \\
\hline Time point 3 & 0.8 & 0.8 & $(0.8 ; 0.9)$ & 0.9 & 0.8 & $(0.7 ; 0.9)$ \\
\hline \multicolumn{7}{|c|}{ Proportion of ANC attendees receiving HIV testing } \\
\hline Baseline & 0.6 & 0.6 & $(0.5 ; 0.7)$ & 0.8 & 0.7 & $(0.5 ; 0.8)$ \\
\hline Time point I & 0.6 & 0.6 & $(0.5 ; 0.7)$ & 0.8 & 0.7 & $(0.6 ; 0.9)$ \\
\hline Time point 2 & 0.8 & 0.8 & $(0.7 ; 0.9)$ & $\mathrm{I}$ & 0.9 & $(0.8 ; 0.9)$ \\
\hline Time point 3 & $\mathrm{I}$ & 0.9 & $(0.9 ; 1)$ & 1 & 0.9 & $(0.9 ; 1)$ \\
\hline \multicolumn{7}{|c|}{ Proportion of those being tested at first ANC visit } \\
\hline Baseline & 0.8 & 0.7 & $(0.6 ; 0.8)$ & 0.8 & 0.7 & $(0.6 ; 0.8)$ \\
\hline Time point I & 0.8 & 0.7 & $(0.6 ; 0.8)$ & 0.8 & 0.7 & $(0.6 ; 0.8)$ \\
\hline Time point 2 & 0.8 & 0.8 & $(0.7 ; 0.8)$ & 0.9 & 0.8 & $(0.8 ; 0.9)$ \\
\hline Time point 3 & 0.8 & 0.8 & $(0.7 ; 0.9)$ & 0.9 & 0.9 & $(0.8 ; 0.9)$ \\
\hline \multicolumn{7}{|c|}{ Proportion of those tested who received their results the same day } \\
\hline Baseline & 0 & 0 & $(0 ; 0)$ & 0 & 0.1 & $(0 ; 0.2)$ \\
\hline Time point I & 0.2 & 0.2 & $(0.1 ; 0.3)$ & 0.1 & 0.2 & $(0.1 ; 0.3)$ \\
\hline Time point 2 & 0.8 & 0.8 & $(0.7 ; 0.9)$ & 1 & 0.7 & $(0.6 ; 0.9)$ \\
\hline Time point 3 & I & 0.9 & $(0.9 ; 1)$ & I & 0.9 & $(0.8 ; 1)$ \\
\hline
\end{tabular}

Table 2:Adjusted random effects logistic models on log odds of achieving $\mathbf{8 0} \%$ testing uptake among pregnant women attending ANC, 80\% ANC attendees being tested for HIV at first visit, and providing test results on the same day to $70 \%$ of those tested by rural and urban health center groups $(2012-2015)$

\begin{tabular}{|c|c|c|c|c|c|c|c|c|c|c|c|c|}
\hline \multirow[t]{2}{*}{ Effect } & \multicolumn{4}{|c|}{$80 \%$ testing uptake } & \multicolumn{4}{|c|}{ Tested at first ANC visit } & \multicolumn{4}{|c|}{ Received same-day test results } \\
\hline & Estimate & SE & DF & t value & Estimate & SE & DF & t value & Estimate & SE & DF & $\mathrm{t}$ value \\
\hline Intercept & -1.72 & 1.43 & 22 & -1.21 & -0.06 & 1.24 & 22 & -0.04 & -8.95 & 3.36 & 22 & -2.67 \\
\hline Time & 0.67 & 0.42 & 153 & 1.58 & 0.6 & 0.39 & 153 & 1.51 & 4.48 & 1.6 & 153 & $2.8^{*}$ \\
\hline Time spline_2 & 1.98 & 1.26 & 153 & 1.57 & -0.38 & 1.02 & 153 & -0.37 & -1.47 & 1.76 & 153 & -0.84 \\
\hline Rural & -3.35 & 1.46 & 153 & $-2.3^{*}$ & -0.42 & 1.04 & 153 & -0.4 & -63.38 & 4.12 & 153 & $-15.4^{*}$ \\
\hline Time*Rural & 1.32 & 0.71 & 153 & $1.85 !$ & -0.6 & 0.56 & 153 & -1.07 & 31.94 & 1.7 & 153 & $18.8 *$ \\
\hline Time spline_2*Rural & -1.82 & 1.77 & 153 & -1.03 & 1.28 & 1.44 & 153 & 0.89 & -32.79 & 0 & 153 & -Infty* \\
\hline $\begin{array}{l}\text { Female literacy less than } \\
93 \%\end{array}$ & 2.2 & 1.05 & 153 & $2.1 *$ & 0.13 & 0.73 & 153 & 0.18 & 2.71 & 1.42 & 153 & 1.91 \\
\hline $\begin{array}{l}\text { No stock, out of HIV } \\
\text { testing kits }\end{array}$ & 0.73 & 0.99 & 153 & 0.73 & 0.01 & 0.8 & 153 & 0.01 & 1.48 & 1.47 & 153 & I \\
\hline $\begin{array}{l}\text { Less than } 4 \text { villages per } \\
\text { provider }\end{array}$ & 0.79 & 0.88 & 153 & 0.9 & 0.07 & 0.72 & 153 & 0.1 & -2.17 & 1.26 & 153 & -1.73 \\
\hline
\end{tabular}

( ${ }^{\text {P-value }}<0.05 ;$ ' $\mathrm{P}$-value $\left.<0.1\right)$ 


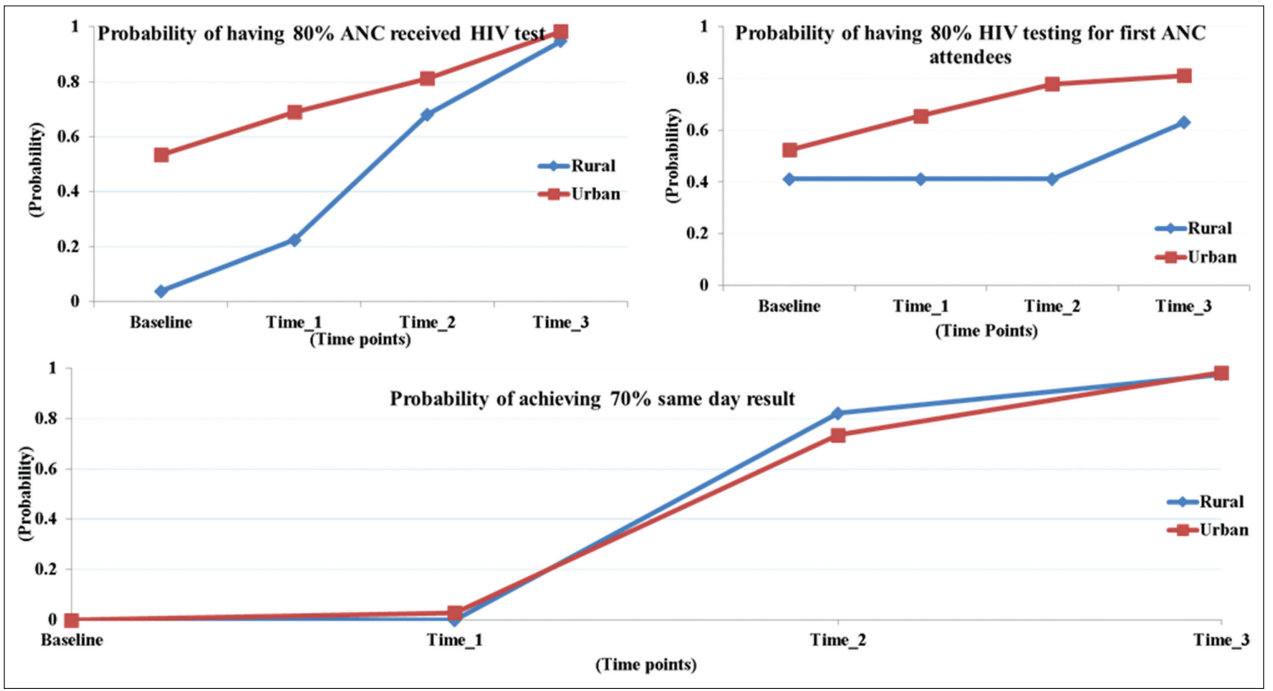

Figure I: $80 \%$ probability for ANC attendees being tested for HIV, $80 \%$ for HIV testing coverage at first ANC visit, and $70 \%$ for receiving same-day results $(2012-2015)$

centers at baseline (P-value $<0.00 \mathrm{I})$, but showed significant improvement throughout the study period. The rate of improvement was $36.42(31.94+4.48) \log$ odds up to time point 2, but slowed down thereafter (Table 2). The progress of providing same-day test results is depicted in terms of probability in Figure I.

\subsection{Service providers' experiences with conducting POC testing}

More than 600 primary health care providers responded to the questionnaire. About half to two-thirds of respondents gave positive or neutral responses. Responses to challenges were compiled and presented according to several identified themes.

\subsection{Logistical supply}

Inadequate availability of HIV test kits and supplemental supplies was cited by $20 \%$ of respondents as a barrier against providing smooth and timely HIV testing services for all ANC attendees at first visit. Frequent changes in test kit brands with different result times and kits with very short shelflives were also cited as a challenge.

\subsubsection{Excessive workload}

As $\mathrm{POC}$ testing was provided together with the essential ANC services, it was of interest to know whether this placed additional burdens on primary care providers. Out of over 600 responses to the questionnaires, $60 \%$ gave neutral responses, while $15 \%$ were very motivated and gave very positive responses such as "POC testing makes ANC more comprehensive" and "the role of the midwife is enhanced." However, $25 \%$ reported additional burdens to their workloads due to providing pre-test information and counseling, performing testing, additional recording and reporting requirements, and discomfort with disclosing positive HIV screening test results.

\subsubsection{Stigma and discrimination}

Although HIV testing of pregnant women has been increasing over time, fear of discrimination still prevents some pregnant women from being tested. Consequently, service providers need to provide repeated counseling so that all pregnant women are tested and receive their results.Another common challenge was obtaining confirmation testing for those with positive results, and for HIV-positive women to enroll in care and receive appropriate treatment. About $25 \%$ of respondents raised the issue of stigma and discrimination as a challenge to providing PMCT services.

\subsubsection{Travel times and transportation costs}

In rural areas, health centers are not available in each village, and one sub-rural health center covers an 
average of five or six villages. Midwives and primary health care providers make day trips to villages to provide ANC, immunization, and other primary health care services. About 15\% of respondents reported that long travel times from health centers to villages left health care providers with inadequate time to provide services, including HIV testing, to all clients during one visit. Long travel times and higher transportation costs deter HIV-positive pregnant women from seeking confirmatory testing and enrolment into care for early treatment.

\subsubsection{Capacity-building requirements}

Counseling was cited by the majority of respondents as the best strategy to get pregnant women tested, to receive confirmation testing, and to receive appropriate treatment for those with a confirmed positive result.About $10 \%$ of respondents requested counseling training for disclosing positive HIV status to spouses and family members. Poor health knowledge among pregnant women was frequently cited as a hindrance to the PMCT program. About $20 \%$ of respondents suggested scaling up community awareness of PMCT services and its advantages in different local dialects and reaching out to the community through various means.

3.4.6. Limited tracking procedures for those lost to follow-up

Respondents experienced limitations for tracking those lost to follow-up. Positive cases sometimes gave false addresses or left town after receiving a positive test result. In addition, there was no proper referral feedback mechanism for those who enrolled for care in the private sector or other national or international organizations providing ART.

\section{Discussion}

\section{I. Discussion}

In this study, three major indicators were used to assess the progress of HIV testing coverage and timeliness of testing and giving results. In general, promising achievements were observed in all indicators by the end of the study period, which was two years after the initiation of the new testing strategy. Further details could be elucidated when comparing the progress of urban and rural health center groups over the study period.

Although both the rural and urban groups demonstrated improvement throughout the study period, rates and patterns of progress varied between the two groups. The urban group had significantly higher log odds of HIV testing coverage and receiving same-day test results at baseline, and had improved early HIV testing from the very beginning, though not statistically significant. The rural group appeared to struggle during the first year of implementation for early HIV testing.

Community-based primary health care in rural areas requires travel by each provider to an average of four to six villages, which is time-consuming, so there was delayed progress in the rural center group. Responses from care providers also indicated a number of challenges that need careful and urgent remedial action.

Heavy workloads, long travel times, and inadequate supply of HIV test kits were common issues reported as major challenges for achieving adequate HIV testing coverage, testing of ANC attendees on the first visit, and same-day results. The entire system of logistical and supply management of the PMCT program should be reviewed and modified to improve conditions in the field. In addition, alternative ways should be found to reduce the workload of primary care providers. Depending on the local context, recruitment and capacity-building of volunteer health workers from each village, such as community health workers and auxiliary midwives, could provide necessary support for primary care providers, especially for health education and group counselling, so that all required tasks can be carried out during the first visit of an ANC attendee.

Stigma and discrimination have been a long-term deterrent for HIV-related activities. ${ }^{14}$ Fear of being stigmatized is a major concern that prevents pregnant women from receiving confirmation testing and enrolling for treatment. Ways to reduce stigma and discrimination should be explored so that the program can reach out to those at risk for HIV infection.

Low levels of health knowledge and awareness of the advantages of PMCT services were reported to 
impede access to the testing and treatment services for PMCT. Communication strategies should include simple and clear behavior change messages on the continuum of care for all pregnant women and family members. Policymakers should consider including HIV testing as one of the essential services for routine ANC, which could help improve HIV testing acceptance among pregnant women and, consequently, early diagnosis and follow-up with necessary care services.

Noticeable improvements of all of the services were observed in both the rural and urban groups throughout the study period. However, much needs to be done to support continuous improvements, and there is a long way to go to achieve universal HIV testing coverage for pregnant women and to achieve an HIV-free generation.

Only $80 \%$ of the estimated numbers of pregnant women in Myanmar were reported to make at least one ANC visit during the study period, and about one-third of those who sought care came during their first trimester. ANC coverage and seeking early ANC among pregnant women needs to be increased. The necessity of improving ANC services is an essential prerequisite for moving towards elimination of paediatric HIV transmission. ${ }^{15}$ This was also raised as a key concern by service providers when an assessment of cascade of services was conducted in Myanmar. ${ }^{16}$

Drug efficacy for prevention of mother-to-child transmission is best when the treatment is started as early as 14 weeks of gestation. ${ }^{17}$ Myanmar adopted the WHO updated guideline in its national PMCT guideline in 2016 to treat all HIV-positive pregnant women as early as possible, regardless of CD4 count. $^{18}$ Our findings also emphasize the need to provide HIV testing for all ANC attendees at the first visit and to give same-day results to maximize numbers of HIV-positive women who will undergo timely diagnosis and enrolment for appropriate treatment. Only about $80 \%$ of pregnant women in Myanmar make at least one ANC visit, and of these, about half do not seek ANC until their second trimester, and less than a sixth during their third trimester. Unless HIV testing and test results occur on the same day, a substantial proportion of HIV-positive pregnant women will not receive appropriate treatment, which could result in HIV transmission to their infants.

A very rapid rollout of $P O C$ testing revealed inadequate skills amongst health care providers in giving disclosure counseling, fear of providing wrong test results, and inadequate privacy for counseling those with positive test results. Improvements and modifications should be undertaken to sustain program improvement. There should be continued visits to the field by training teams to seek solutions to issues such as privacy for HIV testing and counseling for HIV-positive clients, as well as continued professional education plans based on need. Limitations in such support visits were mentioned by some service providers in another study. ${ }^{16}$ The program should set up a standard operating procedure on quality control for HIV screening tests, which could help build the confidence of providers since they are involved in regular quality control schemes and receiving feedback. The quality control plan is also crucial for long-term improvement of the program.

\subsection{Limitations}

There are some limitations to the study that should be noted. The data were extracted from service providers' records and reports, with limited ability to track individual pregnant women completely throughout the cascade of services. Even with these limitations, our findings identified essential elements for improving the effectiveness of PMCT program implementation in the future.

\subsection{Challenges}

Advocacy with supervisors from different levels of the public health department and national AIDS program was a major prerequisite for data collection. Having extracted data from the paper-based records of primary health care providers, careful data completeness and consistency checking was required, and was conducted by the data collection team at study sites. In addition, tremendous efforts for rigorous data verification and triangulation were conducted by the researcher. 


\section{Conclusions and Global Health Implications}

There is an urgent need to take remedial action to ensure continuous availability of testing supplies to primary service delivery points. The program should consider making HIV testing an essential service at the first ANC visit and recruiting local volunteers that could help with community health awareness and health education to reduce the workloads of service providers. Mechanisms for providing continued professional training based on needs and continued mentoring from state, regional and township mentors, and a quality control scheme for HIV testing should be established and implemented. It is firmly believed that with effective implementation of PMCT services fully integrated into primary health care services, great strides can be made towards achieving an HIV-free generation in Myanmar by 2020.

Our study provided evidence that decentralized HIV testing, a community-based strategy, works well, even in rural settings. Adopting communitybased PMCT services can take Myanmar closer to the WHO goal of elimination of mother-to-child transmission of HIV and safeguard sustainability. This strategy would be applicable to other resourcelimited countries.

\section{Compliance with Ethical Standards}

Conflicts of Interest: The authors declare that there is no conflict of interest regarding this study. Financial Disclosure: The authors declare that there is no financial disclosure required for this research. Funding/Support: This study was supported by $\mathrm{NIH} /$ Fogarty International Center training programs D43 TW0000I3 and D43 TW009600 at UCLA. Ethics Approval: This study was carried out with approval from the Institutional Review Board (IRB) of the University of California, Los Angeles (UCLA) and the Myanmar Ethical Review Committee. It was also reviewed and approved by the Department of Public Health, Ministry of Health and Sports, Myanmar. Acknowledgements: This study was funded by NIH/Fogarty International Center D43 TW009600.

\section{Key Messages}

POC testing has improved HIV testing coverage among pregnant women in Myanmar, and reduced the turnaround time for receiving results, which greatly increases the number of pregnant women who know their HIV status.

- Fear of discrimination remains a barrier for pregnant women receiving HIV testing at their first ANC visit. HIV testing with proper counselling is an essential service for the first ANC visit, and increases HIV testing coverage and early diagnosis of HIV among pregnant women. There is a need to establish a mechanism for providing continued professional training based on needs and continued mentoring to ensure quality of HIV testing services by primary care providers

\section{References}

I. Ministry of Health and Sports, Myanmar. National strategic plan on HIV and AIDS 2016-2020 Myanmar. https://aidsdatahub.org/national-strategic-plan-hivand-aids-myanmar-2016-2020-ministry-health-andsports-myanmar-2017.Accessed March 27, 2015.

2. National AIDS Programme. Annual Progress Report on the National HIVIAIDS Response. Nay Pyi Taw, Myanmar: Ministry of Health; $201 \mathrm{I}$.

3. National AIDS Progarm. Global AIDS Response Progress Report, Myanmar, 201I. http:// aidsreportingtool.unaids.org

4. National AIDS Programme. Annual Progress Report on the National HIVIAIDS Response. Nay Pyi Taw, Myanmar: Ministry of Health; 2012.

5. World Health Organization. Consolidated guidelines on the use of antiretroviral drugs for treating and preventing HIV infection: recommendations for a public health approach, $2^{\text {nd }}$ ed. WHO Document Production Services, Geneva, Switzerland: 2016. ISBN 97892 41549684.

6. UNICEF. Asia Pacific Prevention of Parent-To-Child Transmission of HIV Task Force $10^{\text {th }}$ Meeting Report, Bangkok: UNICEF East Asia and Pacific Regional Office;August 2016. ISBN: 978-974-685-I52-7.

7. World Health Organization. Global guidance on criteria and processes for validation of elimination of mother-to-child transmission of HIV and syphilis, $2^{\text {nd }}$

๑ 2019 Global Health and Education Projects, Inc. 
ed. Geneva,WHO; 2017. Licence: CC BY-NC-SA 3.0.

8. AIDS/STD Team. Standard Operating Procedures for HIV Testing and Counselling, Myanmar. Nay Pyi Taw, Myanmar: National AIDS Programme, Ministry of Health and Sports; 2013.

9. Government of Myanmar. The 2014 Myanmar Population and Housing Census - The Union Report - Census ReportVolume 2-B. https://reliefweb. int/report/myanmar/2014-myanmar-population-andhousing-census-union-report-census-report-volume2-b-enmy.Accessed January 2I, 2019.

10. World Health Organization Regional Office for the Western Pacific. Regional framework for the triple elimination of mother-to-child transmission of HIV, hepatitis B and syphilis in Asia and the Pacific, 20I82030. Manila, Philippines; 20 18. Licence: CC BY-NCSA 3.0 IGO.

II. RANDOM.ORG - Integer Generator. https://www. random.org/integers/.Accessed March 12, 2017.

12. Epi Info ${ }^{\mathrm{TM}} \mid \mathrm{CDC}$ https://www.cdc.gov/epiinfo/index. html.

13. Smith T, Smith B. PROC GENMOD with GEE to Analyze Correlated Outcomes Data Using SAS. San Diego, CA: Department of Defense Center for Deployment Health Research, Naval Health Research
Center; 2006. http://www.lexjansen.com/wuss/2006/ tutorials/TUT-Smith.pdf.Accessed June 9, 2015.

14. UNAIDS. People Living with HIV Stigma Index. Yangon, UNAIDS country office for Myanmar; 2016. http:// www.stigmaindex.org/ Accessed January 21, 2019.

I5. IATT, UNICEF, World Health Organization. Toolkit: Expanding and simplifying treatment for pregnant women living with HIV: managing the transition to Option B/B+. Published 2013. https://www.who.int/ hiv/pub/mtct/iatt_optionBplus_toolkit/en/.Accessed March 27, 2015.

16. Mon MM, Htut KM, Oo HN, Lwin SM, Aung MY. Assessment on cascade of PMCT servies received by HIV positive mothers during 2012 and 20I4, Myanmar. Published 2016. In-country report, Ministry of Health and Sports, Myanmar.

17. World Health Organization. PMCT update. pdf. http://www.who.int/hiv/PMCT_update.pdf. Accessed March 27, 2015.

18. National AIDS Programme, Department of Public Health, Ministry of Health and Sports, Myanmar. Guidelines for the Clinical Management of HIV Infection in Myanmar, $5^{\text {th }}$ ed. Published 2017. https:// aidsfree.usaid.gov/sites/default/files/mmr_hiv_ guidelines_2017.pdf.Accessed March 27, 2017. 